\title{
Health Monitoring System for Post-Stroke Management
}

\author{
Elizabeth Adejumo \\ Federal College of Education (Technical) Gombe, Nigeria \\ Email: adejumobola@gmail.com \\ Funmilola Ajala \\ Ladoke Akintola University of Technology, Ogbomoso, Nigeria \\ Email: funfaith2003@yahoo.co.uk
}

Received: 21 September 2018; Accepted: 18 October 2018; Published: 08 January 2019

\begin{abstract}
This paper presents an intelligent health monitoring system for post management of stroke. Fitbit sensor was used to take the reading of four stroke patients in Federal Teaching Hospital, Gombe State, and the vital readings recorded were heartbeat rate, sleeping rate, the number of steps taken, for a period of four weeks. The developed AppFabric, Web service and AppFeedBack synchronized the operation of the sensor, the user mobile device and the medical diagnostic platform. The readings taken by the sensor were made available to the medical experts and the monitoring team using web service. The evaluation of the system in terms of efficiency and reliability using t-Test were $(82.3,85.9)$ and $(1.729133$, $2.093024)$ respectively. The results show that the developed system performed better than the existing manual method for monitoring stroke patients.
\end{abstract}

Index Terms-Stroke, developing countries, WBAN, health monitoring, sensor.

\section{INTRODUCTION AND BACKGROUND}

E-health and telemedicine have proven to be crucial to $21^{\text {st }}$ century health care delivery, and they have been simulated and deployed for several health services such as electronic health records, electronic referral, electronic prescriptions, diabetes patients monitoring and so on $[1,2,3,4,5,6,7]$

Developing countries in Africa are faced with limited medical personnel and inadequate quality health services required to address the many common healthcare problems such as obesity, high blood pressure, irregular heartbeat, stroke, and diabetes which usually demand people to periodically visit their doctors for routine medical checkups [8,9]. This is further compounded by the dilapidating condition of the limited healthcare facilities and the rate of recurrence of chronic diseases prevalent in Africa.
According to a study, cardiovascular diseases- an antecedent to stroke- claimed more lives than cancer yearly. In fact, in 2015 alone stroke was the second leading cause of death after heart disease accounting for $11.8 \%$ of deaths globally [10]. Even though, there is no sufficient data on stoke in Africa, a Global Burden of Disease model-based estimates in [11, 12] showed that the incidence of stroke in Africa and other low- and middle-income country is evidently growing rapidly and that there is a need for an urgent and immediate action.

"Stroke occurs when blood flow to an area of brain is cut off resulting in death of brain cells from oxygen deprivation and the abilities controlled by that area of the brain such as memory and muscle control are lost", this condition requires a medical emergency and treatment must be sought as quickly as possible [13]. Based on a World Health Organization projection, incidences of stroke is expected to be on the high over the next twenty years in developing countries [14]. A typical example of a densely populated developing country in Africa is Nigeria, the prevalence of HIV/AIDS, tuberculosis and malaria in Nigeria and its population is costly and burdensome. The risk of epidemiological transition will further strain the country's resources as a result of increasing stroke prevalence and similar cardiovascular disease. Overall in Africa as of 2015, the annual stroke incidence rates were about 316 per $100 \quad 000$, and prevalence rates was about 981 per 100000 [12]. Currently stroke prevalence in Nigeria is reported to be at 1-14 per 1000 while the 30-day case fatality rate is as high as $40 \%$ [15]. The estimated risk of recurrent stroke in survivors is about $20 \%$ in 5 years, and it was found that risk of first recurrent is 6 times higher than the risk of first-time stroke attack. Generally, the burden of stroke will continue to be a significant healthcare problem unless the incidence of not only first-ever, but also recurrent stroke and its disabling sequels are reduced [16]. 
This study's problem statement is based on the findings that many stroke patients in developing countries faced challenges in securing immediate access to health care after being discharged from hospital, the number are enormous as a result of which most of them suffered secondary attack or death [17, 18]. The most effective way to reduce the burden of recurrent of cerebrovascular disease in the society is prevention, some methods have been proposed to address this [19]. For example, a remote monitoring system called Telestroke was proposed by [16], despite the improvement it brought in the treatment of stroke patient, it is limited in the sense that it does not address the post-discharge management of stroke survivors. Furthermore, medical monitoring systems called Holter monitors have been proposed in which data processing and analysis were performed offline, making such devices impractical for continual monitoring and early detection of medical disorders [20, 21].

The challenges facing developing countries in the area of quality health provisioning and recurrent in case of chronic diseases could be alleviated using Wireless Body Area Network (WBAN) technology which is adopted in this paper. WBAN is one of the latest technologies in health care diagnosis and management consists of various intelligent bio sensors attached on or implanted in the body. It offers promising applications in the area of real time health monitoring to support remote patient in an unobtrusive, reliable and cost-effective manner, thereby providing personalized sustainable services to patients [22]. Therefore, this paper presents the development of an intelligent WBAN health monitoring system for discharged stroke patient in order to prevent and reduce recurrence. To achieve this, a sensor in form of wearable wrist band for monitoring heart beat rate, sleeping rate, as well as distance covered was worn by the stroke patients in this study. The vital signs readings taken were transmitted via Bluetooth of the patient mobile device to the medical personnel, and to the health care monitoring team in charge via Internet. This enabled the medical personnel and the monitoring team in charge to remotely monitor the health condition of the patient. This approach is expected to go a long way in reducing the death rate due to complication after treatment as vital information about the health status of the patient is accessible to the medical personnel in charge and the monitoring team in real time.

\section{RELATED WORK}

Several telestroke system have been proposed in literature and some of the important works are discussed in the section.

Due to the limited health care facilities in rural area, an emergency web based telestroke system was developed in
$[23,24]$ for ischemic stroke called Remote Evaluation of Acute IsCHemic Stroke (REACH) for teleconsultation. The system provides access to anamnesis of stroke patients in order to improve care delivery. The telestroke was found to have a lower mean for onset-to-treatment time than existing systems which helps to facilitate safe administration of thrombolytic therapy to patients within rural communities suffering an acute ischemic stroke.

[25] also presents an emergency telestroke system for acute ischaemic stroke for rural community that connects hospitals with limited neurologist on call to stroke centre. The system was found to reduce inter-hospital transfer and the time of treatment, which justifies the capability of telemedicine to improve quality of health care to stroke patients.

The importance of remote treatment of stroke patients was also established in [26] who in their experiments reported that telestroke can facilitates high rates of intravenous thrombolysis in patients who have had a stroke in community hospitals within a relatively short time frame. Their telestroke system also reduces the number of patients transferred to stroke centre after teleconsultation. A system response time of 12 minutes was also recorded between consultation request and the telemedicine response.

[27] presented the first Italian telestroke for monitoring stages of stroke care, the web-based system used a new interaction model which connects cerebrovascular specialist to emergency unit, and the telestroke was piloted for two years. Though the system did not perform better than the existing REACH, it however confirms the potential of applying telemedicine to a cerebrovascular pathology

[28] carried out a study on the cost effectiveness of a telestroke that connects stroke specialists to remote emergency department physicians and their stroke patients. Incremental cost-effectiveness ratios (ICERs) was produced by combining Quality-adjusted life-years (QALYs) with costs. It was found that, in addition to the benefits of an improved lifelong stroke care, telestroke is cost effective in the long-term,

[29] measured the impact of telestroke on acute stoke care in Catalonia. One the parameters measured for this study was clinical outcome of telestroke patients in a population-based study. It was also reported that telestroke helps to increase the population rate of intravenous thrombolysis, and reduce a large number of interhospital transfers.

[30] proposed prehospital ambulance-based telestroke as a way to improve stroke diagnosis and timeliness of treatment. The ambulance was equipped with a mobile telemedicine system to perform remote stroke assessments. Simulation of emergency scenarios and interviews with users were used to access the feasibility of the system. The usability scores of the users were however found to vary from their formative feedback 
This study will simulate Information and Information and Communication Technologies approach in telestroke using WBAN.

\section{MATERIAL AND METHODS}

The approach used in this research work were design, experimentation and implementation approach. The scientific approaches employed to realize the research objectives follows the structure in Fig. 1. The developed health monitoring system comprises three tiers - the users' layer, the sync layer and the medical diagnostic layer. An experimental framework for health monitoring system is designed to take readings from the stroke patients. Since clinical indicators are commonly used in health care to represent constraints and optimize performance; system performance was evaluated using efficiency and reliability.

\section{A. Data Acquisition}

The vital signs used for this research were heartbeat rate, sleeping rate and number of steps taken by the patients. These parameters were used to examine four stroke patients in Federal Teaching Hospital Gombe State, Nigeria. The data acquisition periods was two weeks, with recording taking place once a day. In addition, the research collected the same set of patients' data using existing manual method.

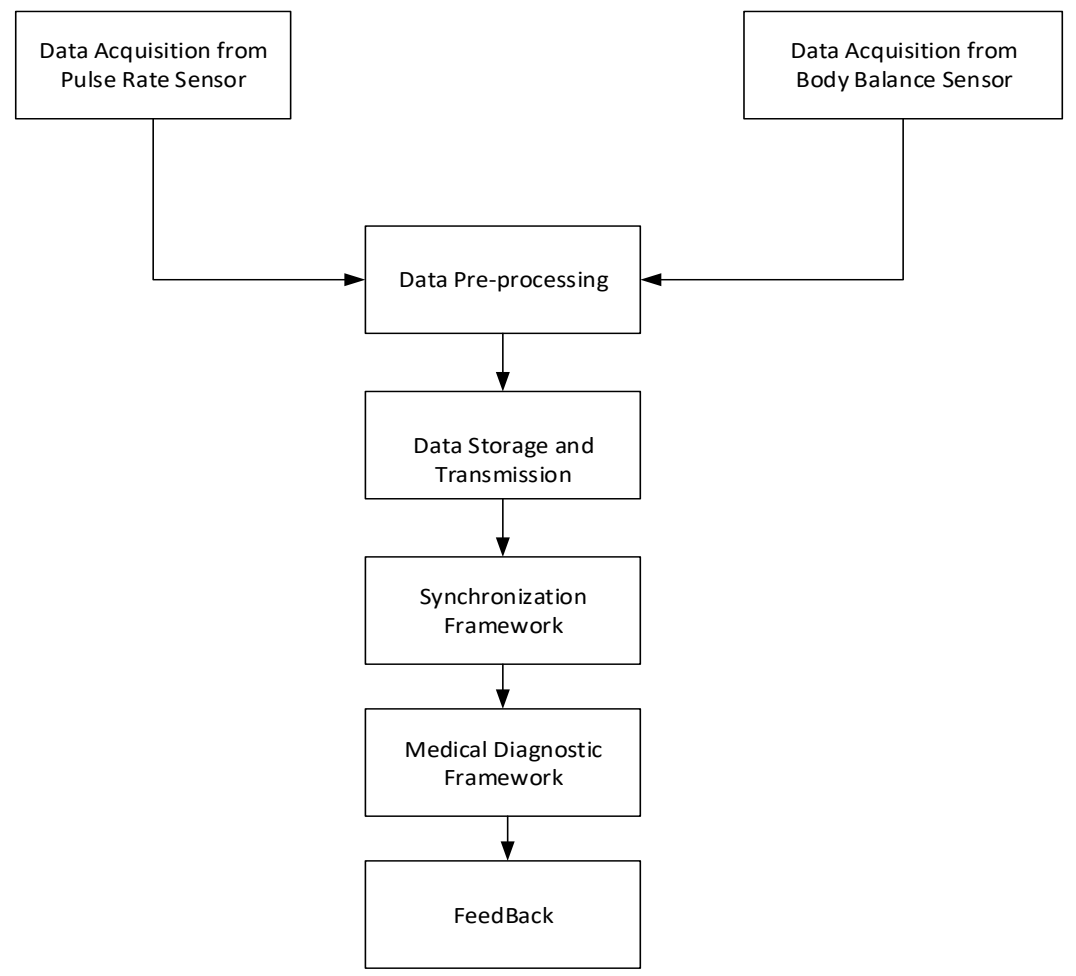

Fig.1. Block diagram of the developed system

\section{B. System Architecture}

The architectural framework in Fig. 2 describes the entire process of the implemented system. The application framework was broadly divided into three: i) User Framework ii) Synchronization Framework iii) Medical Diagnostic Framework.

\section{System Architecture}

User Framework comprises:

i. Wearable Sensor (Fit bit): This was used to measure the heart beat rate, sleeping rate and steps or distance covered. ii. Mobile Phone: This was the device used in this research to house the lightweight database management system used (SQLite). This database was built to store the readings of the basic vital signs considered in this work directly from the wearable sensor. The mobile application that interprets the readings from the sensor was also installed on the mobile phone.

iii. SQLite Database: SQLite is a software library that implements a self-contained, zero-configuration, transactional SQL database engine. This was installed with the mobile app built for user framework

iv. Mobile Communication Signal 


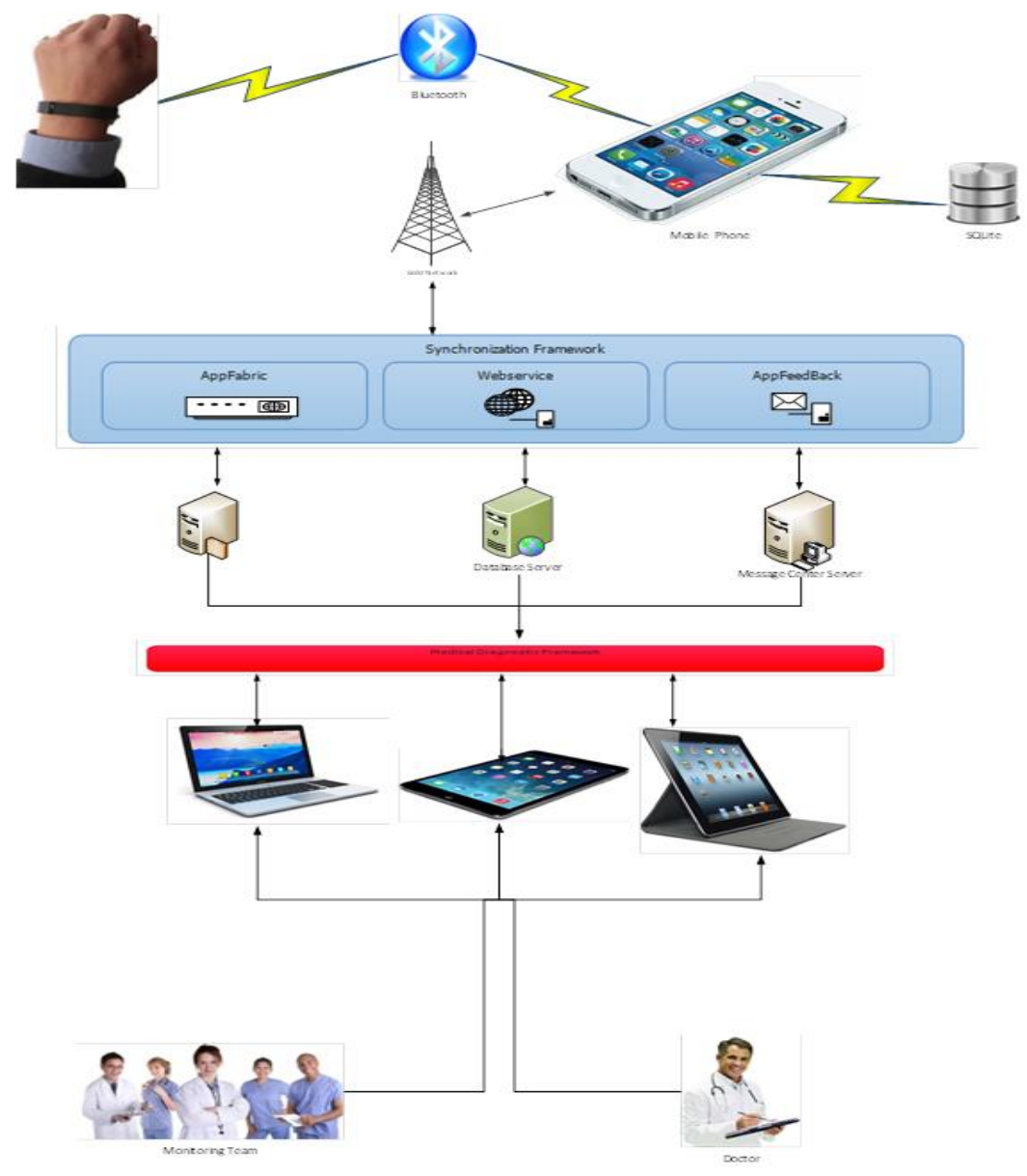

Fig.2. Architectural framework of the developed health monitoring system

\section{System Architecture}

Sync Framework is a comprehensive synchronization platform that enables collaboration and offline access for applications, services, and devices. This framework consists of intermediate resources to transfer data to and from the remote patient to the medical experts. The components in this model are:

i. Database Server: This server was used to store all the remote patients' record, while the SQLite stored only the pieces of information of a specific user, the database server stored all the records of the entire patients. The database management system used in this research was Microsoft SQL Server 2012. The medical diagnostic framework used the data stored here to make clinical decisions.

ii. Web Server: The user framework and medical diagnostic framework cannot communicate to the database directly for security purpose and Separation of Concern in software engineering, therefore a web service was developed to administer interoperability, because the mobile framework was built with proprietary Java programming language and the medical diagnostic framework was built using C\#. The web service was stored on the web server (Internet Information Service) in order to be accessible via hypertext transfer protocol which interprets the Simple Object Access Protocol message to any of the application.

iii. AppFabric: This server manages Remote Method Invocation to trigger the web services and the message feedback regularization framework. The security protocol used to protect applications under the supervision of AppFabric is called Code Access Security which is Microsoft's solution to prevent malicious code from performing privileged actions. When the Common Language Runtime loads an assembly, it will obtain evidence for the assembly and use this to identify the code group that the assembly belongs to.

iv. AppFeedBack: This server was used to manage feedback to the remote stroke patient. The applications in this framework were developed to manage feedback in both synchronous and asynchronous mode. The synchronous mode would send the feedback to the remote stroke patients via SMS immediately the data from the patients' phone got to the database server in the synchronization framework. When no medical personnel are available to trigger feedback, the application will be queued on the server so as to receive attention when the personnel are available. The queue system used in this research was Round Robin with quantum time of two so as to attend to all the patients with equal chances 


\section{E. Medical Diagnostic Framework}

This framework is composed of the team of medical expert purposely charged for this responsibility; they made use of laptops, iPhones and tablets to access information from the synchronization framework. Shift management was also considered for the design in this framework. The components of the framework are:

i. Wearable Sensor: This component represents the Wristband Sensor for taking readings

ii. Bluetooth: This represents the Bluetooth component embedded inside the phone for communicating with the sensor.

iii. SQLite: This is a lightweight database used to store the readings from the sensor; this was installed on the phone to manage records read from the device via the Bluetooth.

iv. Mobile phone: This component has both the Bluetooth and the SQLite database. It is also programmed to synchronize with the synchronization framework.

v. Medical Diagnostic Platform: This component was used to manage the Medical Diagnostic Framework.

vi. Synchronization: The component has the capability to process data transfer from the User and the Medical Diagnostic Platform

vii. Web Server: The web server of choice was the Internet Information Service (IIS) server

viii. Database Server: Database server is the data repository for the readings of all the patients' after synchronization. The Database Management System used for this research is Microsoft SQL Server 2012 Enterprise Version.

ix. Laptop: This is the work station for the medical personnel to access stored record from the main stream database and processed it to make decision on either the feedback or for access.

x. IPad: This is used for mobile access ubiquitously.

The diagram shown in Fig. 3 describes the sequence of the application from User platform to the Sync Framework. Fig. 4 depicts a Use Case Diagram for Diagnostic Medical Platform (DMP) actor which performed the following. i. ConnecttoSync Engine ii. Synchronized iii. Manageshift iv. ManageQueue v. ProcessFieldBack for both synchronous and Asynchronous mode.

\section{F. Performance Evaluation of the Developed System.}

The metrics used for evaluating the performance of the system were efficiency and reliability using Statistical Package for Social Sciences and Excel as the statistical tools. The indices used to determine the metrics are: BPM1(heart beat for the developed system), BPM2 (heart beat for the existing system), mean value (efficiency), mean difference (reliability) and variance of reliability

\section{RESUltS AND DisCUSSIONS}

The developed health monitoring system for post management of stroke survivors monitors the heart beat per minute, sleeping rate and number of steps taken. This is as a result of the findings that showed that these parameters are directly tied to a number of cardiovascular risk factors. The developed system has three platforms which are: i. Mobile Application ii. Web iii. Short Message Service platform

The interface shown in Fig. 5 shows the readings taken from a mobile device of the remote patient that measured the total number of steps taken by the patient. This view is made possible on the user's mobile phone so as to personally check the readings at any time, and at the same time the readings were stored on the database of the device and sent to the medical diagnostic framework on weekly basis via the Sync framework. Also, the interface shown in Fig. 6 is used to measure the sleeping rate of the patient because lack of sleep can also lead to an increase in an unhealthy protein called C-Reactive Protein (CRP), which is directly tied to a number of cardiovascular and heart disease risk factors. This build up in CRP is a sign of increased stress in the body, something that might be ignored in the day-to-day grind but is a very real factor when it comes to heart disease. Thus, measuring the rate of sleeping was imperative. Fig. 7 records the rate of heart beat of the remote patient. Heart rate, or heart pulse, is the speed of the heartbeat measured by the number of contractions of the heart per unit of time typically beats per minute (bpm). The heart rate is rhythmically generated by the sinoatrial node.

The normal resting adult human heart rate ranges from 60-100 bpm. Tachycardia is a fast heart rate, defined as above $100 \mathrm{bpm}$ at rest. Bradycardia is a slow heart rate, defined as below $60 \mathrm{bpm}$ at rest. During sleep a slow heartbeat with rates around 40-50 bpm is common and is considered normal. When the heart is not beating in a regular pattern, this is referred to as an arrhythmia. In measuring the heart beat both the sensor and the manual method were used and these represent BPM1 and BPM2 respectively. Fig. 8 shows the web interface for medical diagnostic framework. Fig. 9 shows a sample message sent to a remote patient from the medical expert. Fig. 10 shows the web service used at the synchronization framework. The heartbeat (BMP), blood pressure (BP), for each of the four patients' readings using sensor and manual methods are presented are shown in Table 1, Table 2, Table 3 and Table 4, together with sleeping rate and number of steps readings. Using pulse rate as the parameter for evaluation, the analysis of the developed and existing system in terms of efficiency and reliability is shown in Table 5. From the t-Test results, the developed system has better values for efficiency and reliability compare to the existing system (lower value is considered because the parameter measured is pulse rate). Also, the critical value taken using t-Test gave a value of 
0.037181 which is lower than the computed. This also affirms the reliability of the developed system is estimates at $\mathrm{P} \leqslant 0.05$. Statistically, there is $95 \%$ confidence that there exist differences between the readings taken by the sensor versus the manual method. The sensor can read an average of 0.0 .4592 to 6.4592 less that the manual method. This is because data are captured and transmitted accurately and with lesser errors in real time. This implies that in addition supporting lifelong care for post stroke patients cheaply, WBAN in telestroke is capable of performing with better accuracy than the manual method in developing countries.

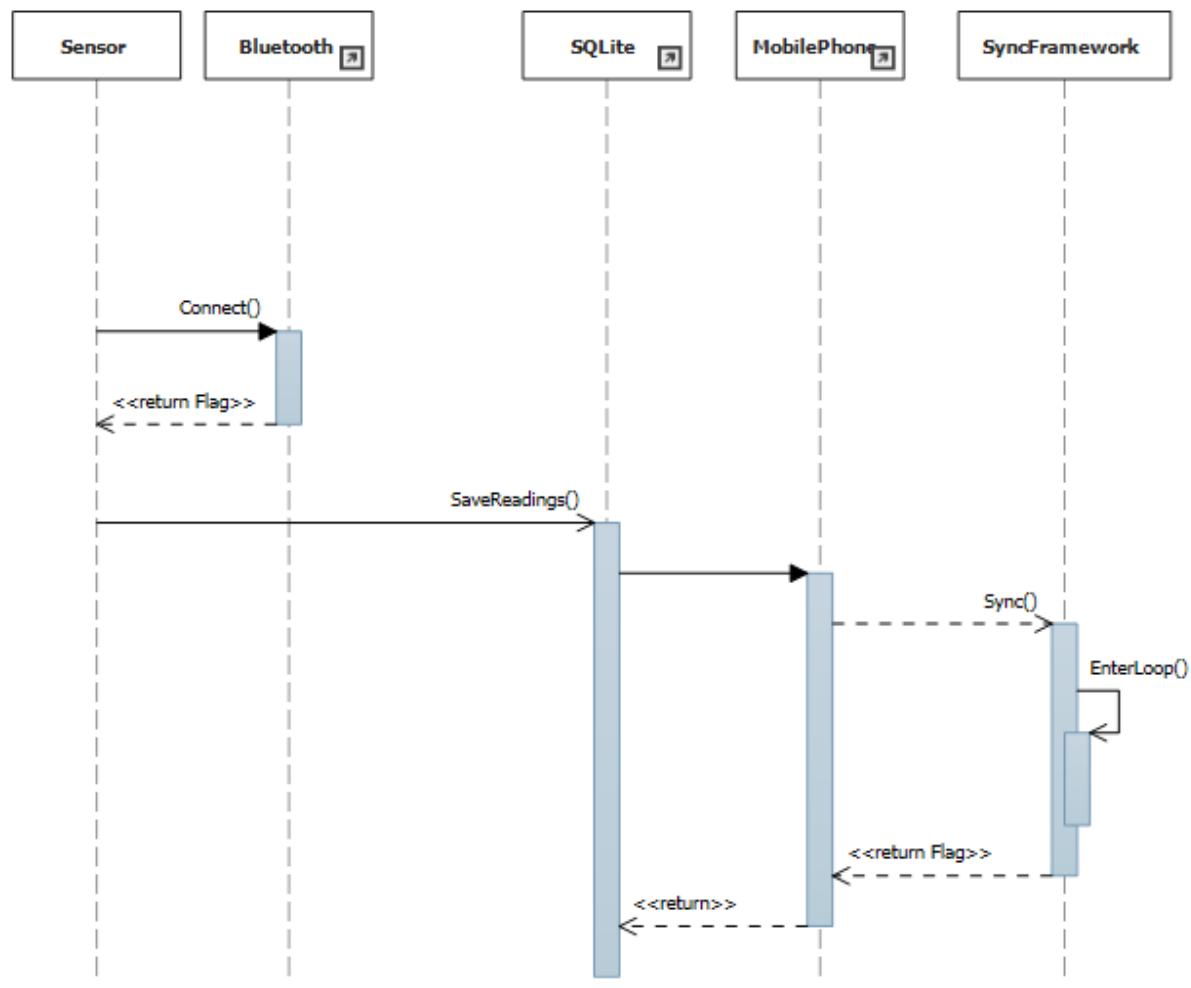

Fig.3. Sequence diagram for the developed health monitoring system.

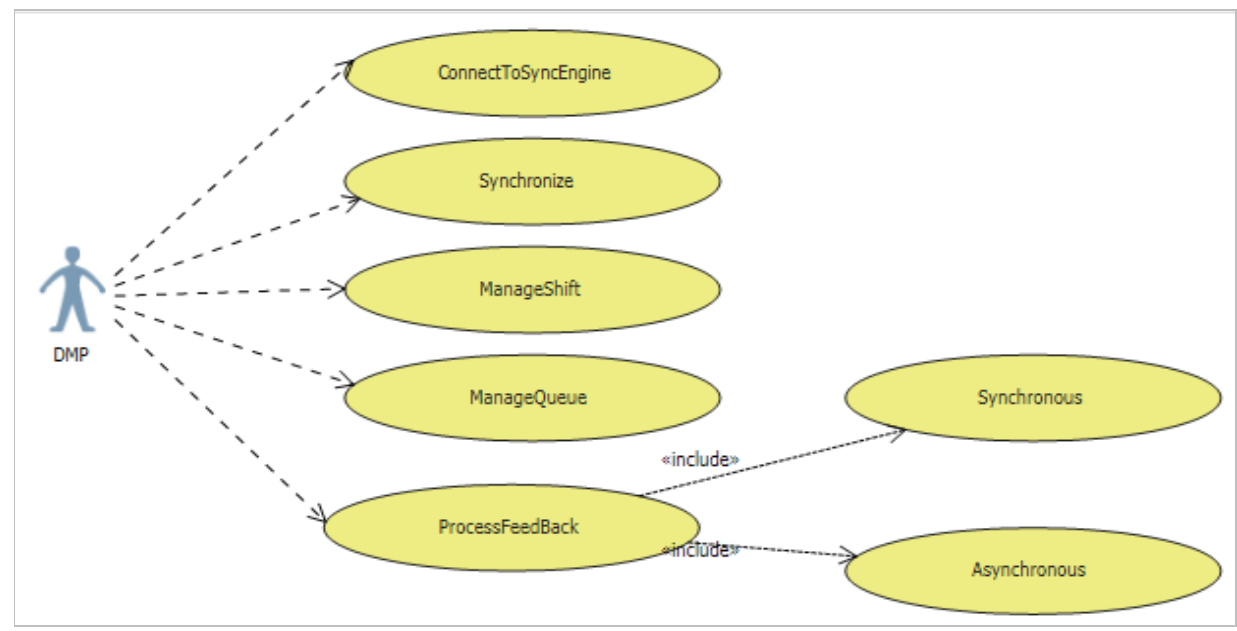

Fig.4. Use case diagrams for the diagnostic medical platform 


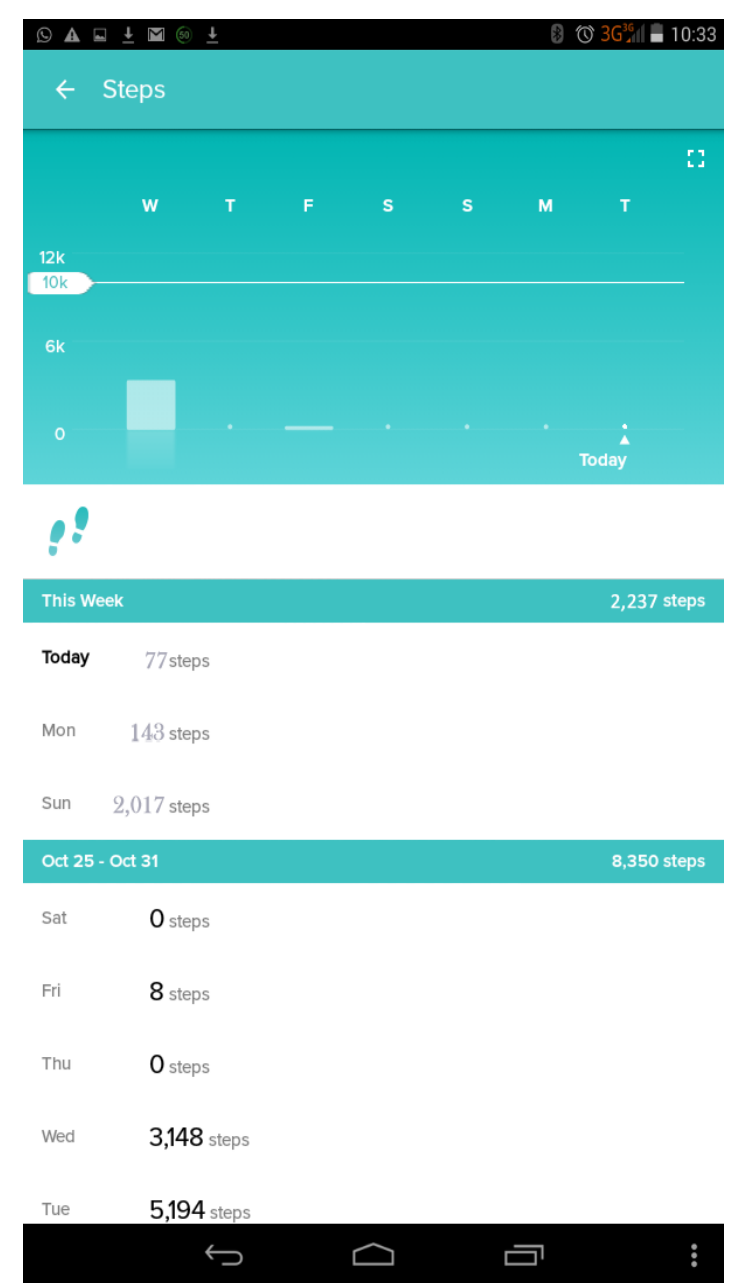

Fig.5. Mobile interface indicating number of steps
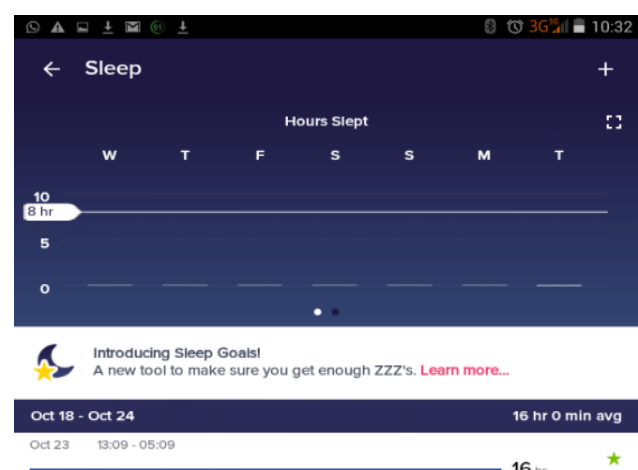

$\hookrightarrow$

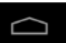

ஏ

Fig.6. Mobile interface showing sleeping rate

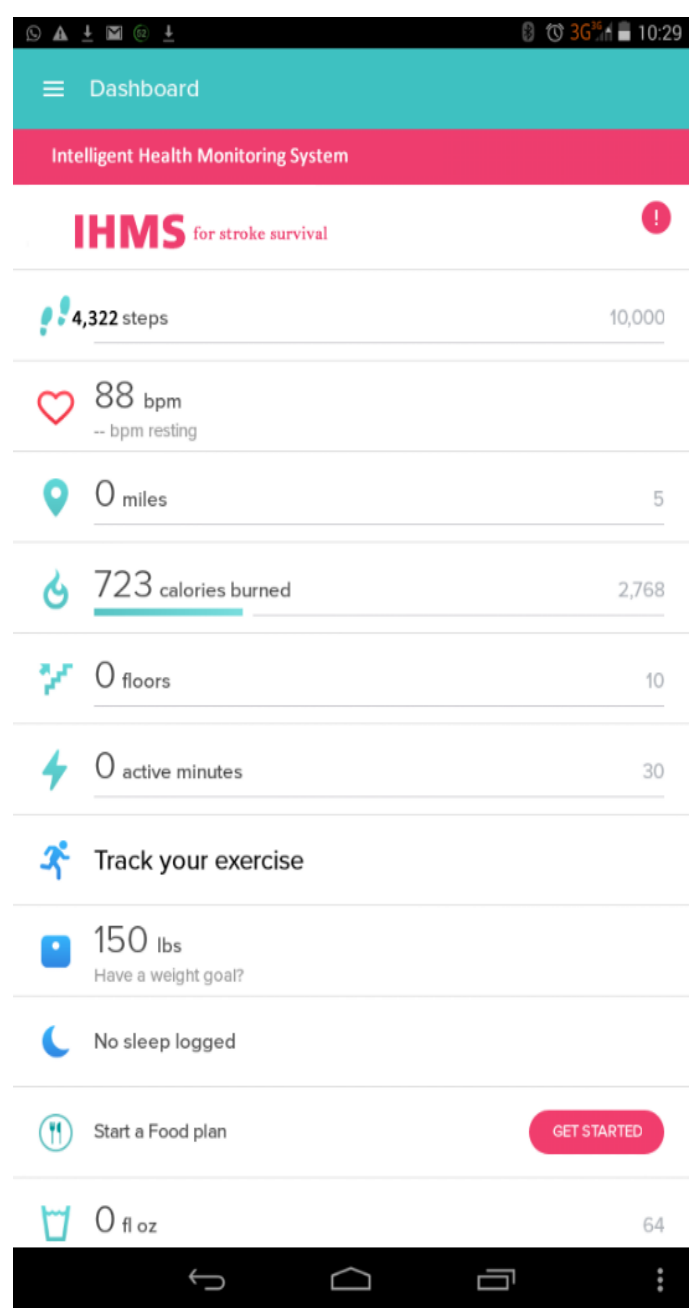

Fig.7. Mobile interface showing heart beat per minute

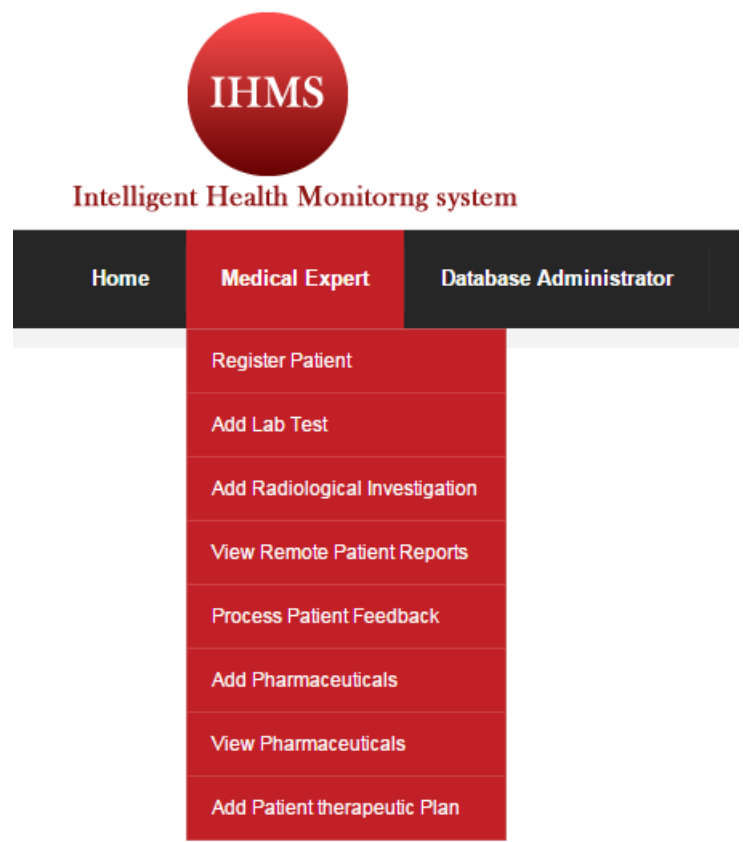

Fig.8. Web interface showing medical expert platform 


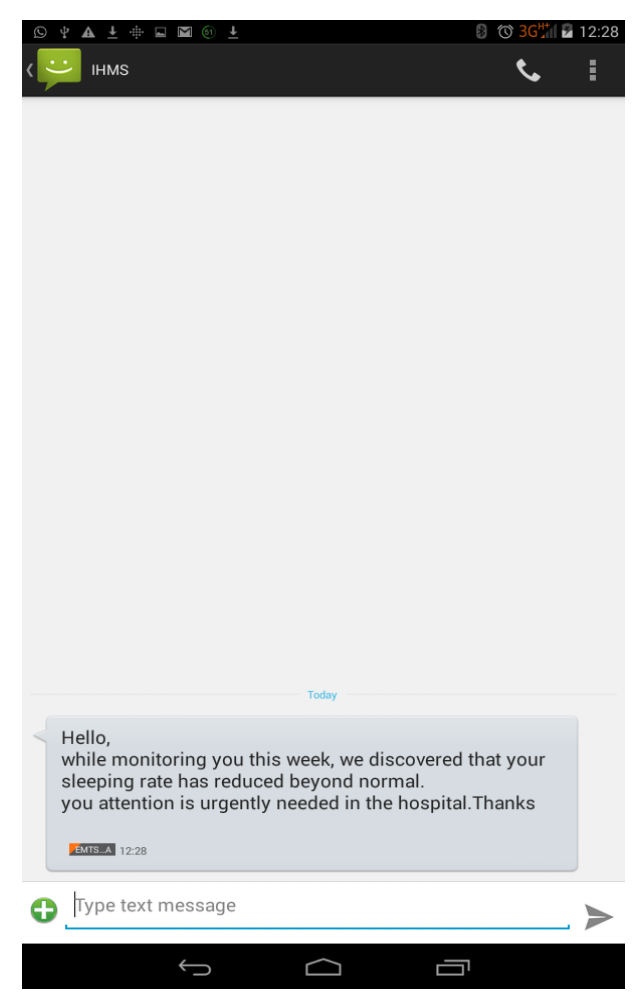

\section{Service}

The following operations are supported, For a formal defintion, plesse review the Service Description,

- ConnectToRemotelser

- GetAllRemotestrokePatients

- GetMedicalPersonelOnSchedule

- Helloworld

1 InterpreteECG

- ProcessAppFabric

- ProcessFeedBack

- TransferSiqual

Fig.10. Interface showing the web service

Fig.9. Interface showing feedback SMS

Table 1. BMP readings taken from patient one

\begin{tabular}{|c|c|c|c|c|c|c|}
\hline Day & BPM1 & BPM2 & BP1 & BP2 & $\begin{array}{c}\text { Sleeping } \\
\text { Rate }\end{array}$ & $\begin{array}{c}\text { No of } \\
\text { steps }\end{array}$ \\
\hline Monday & 84 & 84 & $220 / 120$ & $230 / 140$ & 12 & 883 \\
\hline Tuesday & 88 & 74 & $200 / 110$ & $220 / 126$ & 16 & 1021 \\
\hline Wednesday & 90 & 72 & $260 / 170$ & $260 / 170$ & 8 & 576 \\
\hline Thursday & 78 & 80 & $230 / 140$ & $246 / 160$ & 10 & 323 \\
\hline Friday & 80 & 82 & $246 / 150$ & $248 / 150$ & 14 & 1708 \\
\hline
\end{tabular}

Table 2. BMP readings taken from patient two

\begin{tabular}{|c|c|c|c|c|c|c|}
\hline Day & BPM1 & BPM2 & BP1 & BP2 & $\begin{array}{c}\text { Sleeping } \\
\text { Rate }\end{array}$ & $\begin{array}{c}\text { No of } \\
\text { steps }\end{array}$ \\
\hline Monday & 94 & 86 & $210 / 110$ & $200 / 110$ & 16 & 512 \\
\hline Tuesday & 92 & 80 & $210 / 114$ & $210 / 114$ & 14 & 504 \\
\hline Wednesday & 80 & 82 & $240 / 142$ & $238 / 146$ & 12 & 821 \\
\hline Thursday & 90 & 80 & $196 / 110$ & $200 / 120$ & 13 & 156 \\
\hline Friday & 88 & 86 & $210 / 100$ & $200 / 110$ & 14 & 1210 \\
\hline
\end{tabular}

Table 3. BMP readings taken from patient three

\begin{tabular}{|c|c|c|c|c|c|c|}
\hline Day & BPM1 & BPM2 & BP1 & BP2 & $\begin{array}{c}\text { Sleeping } \\
\text { Rate }\end{array}$ & $\begin{array}{c}\text { No of } \\
\text { steps }\end{array}$ \\
\hline Monday & 88 & 80 & $196 / 104$ & $200 / 100$ & 10 & 748 \\
\hline Tuesday & 90 & 86 & $194 / 100$ & $196 / 106$ & 11 & 127 \\
\hline Wednesday & 80 & 80 & $210 / 124$ & $216 / 130$ & 6 & 476 \\
\hline Thursday & 90 & 88 & $190 / 110$ & $184 / 106$ & 8 & 867 \\
\hline Friday & 94 & 82 & $180 / 100$ & $176 / 98$ & 9 & 589 \\
\hline
\end{tabular}

Table 4. BMP readings taken from patient four

\begin{tabular}{|c|c|c|c|c|c|c|}
\hline Day & BPM1 & BPM2 & BP1 & BP2 & $\begin{array}{c}\text { Sleeping } \\
\text { Rate }\end{array}$ & $\begin{array}{c}\text { No of } \\
\text { steps }\end{array}$ \\
\hline Monday & 74 & 88 & $186 / 102$ & $186 / 104$ & 6 & 1431 \\
\hline Tuesday & 86 & 84 & $182 / 100$ & $180 / 100$ & 8 & 652 \\
\hline Wednesday & 78 & 86 & $188 / 104$ & $190 / 110$ & 13 & 902 \\
\hline Thursday & 82 & 90 & $162 / 100$ & $160 / 100$ & 7 & 790 \\
\hline Friday & 92 & 80 & $150 / 100$ & $150 / 100$ & 10 & 806 \\
\hline
\end{tabular}


Table 5. Analysis of the developed system using t- test paired two sample for means

\begin{tabular}{|c|c|c|}
\hline & BPM1 & BPM2 \\
\hline Mean & 82.3 & 85.9 \\
\hline Variance & 19.06316 & 35.56842 \\
\hline Observations & 20 & \\
\hline Pearson Correlation & 0.57807 & \\
\hline $\begin{array}{c}\text { Hypothesized Mean } \\
\text { Difference }\end{array}$ & 0 & \\
\hline Df & 19 & \\
\hline $\mathrm{P}(\mathrm{T} \leq \mathrm{t})$ one-tail & 0.018591 & \\
\hline $\mathrm{t}$ Critical one-tail & 1.729183 & \\
\hline $\mathrm{P}(\mathrm{T} \leq \mathrm{t})$ two-tail & 0.037181 & \\
\hline $\mathrm{t}$ Critical two-tail & 2.093024 & \\
\hline
\end{tabular}

\section{CONCLUSION}

This paper developed a prototype real time monitoring system for stroke survivor in remote environment and was made available to medical personnel via Web, Mobile and SMS platform using WBAN technology. It is an attractive system of delivering services to stroke survivor without need for both the patient and health care personnel to be in the same location at the same time. It has a major role in providing remote surveillance to patients with chronic conditions, fills a service gap among those who have limited access to expert care and provides important clinical information to the health provider in real time. The developed system has three layers which are user, synchronization and medical diagnostic layers. Each of these represents a node. Nodes are devices or data point on a larger network. This research work does not consider the load failure at nodes, thus subsequent research may need to study this.

\section{ACKNOWLEDGMENT}

The authors wish to thank the staff and volunteers at Federal Teaching Hospital Gombe State for supporting this work.

\section{REFERENCES}

[1] A. Omotosho, U. Asanga, and A. Fakorede. "Electronic Prescription System for Pediatricians", European Scientific Journal, 13, no. 18, (2017): 426 - 437,

[2] A. Omotosho, J. Emuoyibofarhe, and C. Meinel. "Ensuring patients' privacy in a cryptographic-based-electronic health records using bio-cryptography." International Journal of Electronic Healthcare 9, no. 4 (2017): 227-254.

[3] A. Omotosho., J. Emuoyibofarhe, and A. O. Oke. "Securing Private Keys in Electronic Health Records Using Session-Based Hierarchical Key Encryption." Journal of Applied Security Research 12, no. 4 (2017): 463-477.

[4] A. Omotosho, O., Adegbola, and A. Adebo. "A Patientbased Hospital Referral Decision Support System." International Journal of Computer Application 155, no. 10 (2016): $38-43$

[5] A. Omotosho, and O. J Emuoyibofarhe "A Secure Intelligent Decision Support System for Prescribing Medication." Computing, Information Systems and
Development Informatics Journal 3, no.3 (2012): 9-18

[6] O.M Olaniyi, A. Omotosho, S. A. Bashir, O.M. Olaniyan, and A.D. Ojiakor, "Multifaceted E-health System for Monitoring Diabetes Patient". In proceedings of the Fourth International Conference on Mobile e-Services and Workshop on IT Systems Engineering (ICOMeS 2012) , Lautech, Ogbomoso, Nigeria. Oct. 16 - 17, 2012. 4(2012): $96-106$.

[7] A. Omotosho, O. M. Olaniyi, O. J. Emuoyibofarhe, and O. B. Osobu "Electronic Medication Prescribing Support System for Diagnosing Tropical Diseases". In proceedings of the Fourth International Conference on Mobile e-Services and Workshop on IT Systems Engineering (ICOMeS 2012), Lautech, Ogbomoso, Nigeria. Oct. 16 - 17, 2012. 4(2012): 57 - 66.

[8] P. Turner, "Biosensors: sense and sensibility." Chemical Society Reviews 42, no. 8 (2013): 3184-3196.

[9] M. Kifle, Mengistu, V. W. Mbarika, C. Tsuma, D. Wilkerson, and J. Tan. "A TeleMedicine transfer model for sub-Saharan Africa." In Hawaii International Conference on System Sciences, Proceedings of the 41st Annual, pp. 244-244. IEEE, 2008.

[10] American Stroke Association, Heart Disease and Stroke Statistics $2018 \quad$ At-a-Glance (2018) https://healthmetrics.heart.org/wpcontent/uploads/2018/02/At-A-Glance-Heart-Diseaseand-Stroke-Statistics-2018.pdf

[11] A. Moran, M. Forouzanfar, U. Sampson, S. Chugh, V. Feigin, and G. Mensah, "The epidemiology of cardiovascular diseases in sub-Saharan Africa: the global burden of diseases, injuries and risk factors 2010 study." Progress in cardiovascular diseases 56, no. 3 (2013): 234-239.

[12] M. O. Owolabi, O. Arulogun, S. Melikam, A. M. Adeoye, S. Akarolo-Anthony, R. Akinyemi, D. Arnett, H. Tiwari, M. Gebregziabher, C. Jenkins, and D. Lackland, "The burden of stroke in Africa: a glance at the present and a glimpse into the future." Cardiovascular journal of Africa 26, no. 2 H3Africa Suppl (2015): S27.

[13] National Stroke Association, "What is stroke?" (2018). Available at http://www.stroke.org/understandstroke/what-stroke

[14] World Health Organization, "International statistical classification of diseases and related health problems", Tenth Revision vol 2, Geneva, (2008), Available at http://www.who.int/classifications/icd/ICD10 Volume2_en_2008.pdf

[15] K. W. Wahab, "The burden of stroke in Nigeria." International journal of stroke 3, no. 4 (2008): 290-292. 
[16] J. Joubert, E. Medeiros de Bustos, P. Decavel, J. L. Chopard, L. B. Joubert, and T. Moulin. "Telestroke for the long-term management of risk factors in stroke survivors." In Telemedicine Techniques and Applications. InTech, 2011.

[17] J. Joubert, L. Joubert, C. Reid, D. Barton, T. Cumming, P. Mitchell, M. House et al. "The positive effect of integrated care on depressive symptoms in stroke survivors." Cerebrovascular diseases 26, no. 2 (2008): 199-205.

[18] G. J. Hankey, "Secondary stroke prevention." The Lancet Neurology 13, no. 2 (2014): 178-194.

[19] P. B. Gorelick, "Stroke prevention: windows of opportunity and failed expectations?." Neuroepidemiology 16, no. 4 (1997): 163-173.

[20] M. Aminian, and H. R Naji. "A hospital healthcare monitoring system using wireless sensor networks." $J$. Health Med. Inform 4, no. 02 (2013): 121.

[21] M. S. Sudha, and R. Selvakumar. "A Hospital Healthcare Monitoring System Using Wireless Sensor Networks." architecture 5, no. 05 (2018).

[22] G. K. Ragesh, and K. Baskaran. "A survey on futuristic health care system: WBANs." Procedia Engineering 30 (2012): 889-896.

[23] J. A. Switzer, C. Hall, H. Gross, J. Waller, F. T. Nichols, S, Wang, R. J Adams, \& D. C. Hess, D.C. "A web-based telestroke system facilitates rapid treatment of acute ischemic stroke patients in rural emergency departments." The Journal of emergency medicine 36, no. 1 (2009): 1218.

[24] D. C. Hess, S. Wang, H. Gross, F. T. Nichols, C. E. Hall, \& R. J. Adams. "Telestroke: extending stroke expertise into underserved areas." The Lancet Neurology 5, no. 3 (2006): 275-278

[25] A. Pedragosa, J. Alvarez-Sabin, C. A. Molina, C. Sanclemente, M. C. Martín, F. Alonso, \& M. Ribo, M. (2009). "Impact of a telemedicine system on acute stroke care in a community hospital." Journal of telemedicine and telecare 15, no. 5 (2009): 260-263.

[26] N. Chalouhi, J. A. Dressler, E. S. Kunkel, R. Dalyai, P. Jabbour, L. F. Gonzalez, R. M. Starke, A. S. Dumont, R. Rosenwasser, \& S. Tjoumakaris. "Intravenous tissue plasminogen activator administration in community hospitals facilitated by telestroke service." Neurosurgery 73, no. 4 (2013): 667-672.
[27] L. Nardetto, C. Dario, S. Tonello, M. C. Brunelli, M. Lisiero, M. G. Carraro, C. Saccavini, G. Scannapieco \& B Giometto. "A one-to-one telestroke network: the first Italian study of a web-based telemedicine system for thrombolysis delivery and patient monitoring." Neurological Sciences 37, no. 5 (2016): 725-730.

[28] R. E. Nelson, G. M. Saltzman, E. J. Skalabrin, B. M. Demaerschalk, \& J. J. Majersik . "The cost-effectiveness of telestroke in the treatment of acute ischemic stroke." Neurology 77, no. 17 (2011): 1590-1598.

[29] E. López-Cancio, M. Ribó, P. Cardona, J. Serena, F. Purroy, E, Palomeras, J. M. Aragonès, D. Cocho, M. Garcés, E. Puiggròs \& I, Soteras. "Telestroke in Catalonia: Increasing Thrombolysis Rate and Avoiding Interhospital Transfers." Cerebrovascular Diseases 46, no. 1-2 (2018): 66-71.

[30] S.N.C. Smith, P. C. Brown, K. H. Waits, J. S. Wong, M. S Bhatti, Q. Toqeer, J. V. Ricks, M. L. Stockner, T. Habtamu, J. Seelam, \& R. C. Britt. "Development and Evaluation of a User-Centered Mobile Telestroke Platform." Telemedicine Journal and E-health (2018).

\section{Authors' Profiles}

Elizabeth Adejumo is a lecture at the Department of Computer Science in Federal College of Education (Technical) Gombe. She holds an MSc in Computer Science from Ladoke Akintola University of Technology. Her research areas are ehealth and telemedicine.

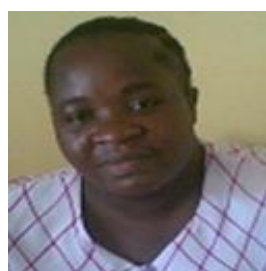

Funmilola Ajala is a Senior Lecturer at the Department of Computer Science and Engineering in Ladoke Akintola University of Technology. She holds a $\mathrm{PhD}$ in Computer from Ladoke Akintola University of Technology. She has published in several international journals and conferences in the field of ehealth and telemedicine.

How to cite this paper: Elizabeth Adejumo, Funmilola Ajala, "Health Monitoring System for Post-Stroke Management", International Journal of Information Engineering and Electronic Business(IJIEEB), Vol.11, No.1, pp. 110, 2019. DOI: 10.5815/ijieeb.2019.01.01 